\title{
A RELAÇÃO ENTRE A PERCEPÇÃO DE PRÁTICAS DE RESPONSABILIDADE SOCIAL CORPORATIVA E A INTENÇÃO DE ROTATIVIDADE DOS PROFISSIONAIS.
}

\author{
Kátia Cyrlene de Araujo Vasconcelos* \\ katia.vasconcelos@oi.com.br \\ Annor da Silva Junior* \\ annor.silva@ufes.br \\ Adelson Pereira do Nascimento* \\ adelsonpn@gmail.com \\ Vania Maria Goulart* \\ vania@selecta-es.com.br \\ *Universidade Federal do Espírito Santo
}

http://dx.doi.org/10.1590/1413-2311.01116.62787

Recebido em 12/09/2014

Aprovado em 23/08/2016

Disponibilizado em 31/01/2017

Avaliado pelo sistema "double blind review"

Revista Eletrônica de Administração

Editora-chefe: Aurora Zen

ISSN 1413-2311 (versão "on line")

Editada pela Escola de Administração da Universidade Federal do Rio Grande do Sul.

Periodicidade: Quadrimestral

Sistema requerido: Adobe Acrobat Reader

\section{RESUMO}

Analisou-se neste artigo a relação entre a percepção de práticas de responsabilidade social corporativa (RSCORP) e a intenção de rotatividade (IR) de profissionais brasileiros de diferentes Estados e setores econômicos. Coletou-se dados por meio de questionário baseado nas escalas de percepção de práticas de responsabilidade social corporativa (TURKEY, 2009b) e escala de intenção de rotatividade (SIQUEIRA et al., 2012) junto a 100 profissionais. Os dados foram analisados por meio de modelagem em equações estruturais utilizando o software Smart PLS 3. A síntese dos resultados indica que a percepção de práticas de responsabilidade social corporativa influencia negativamente a intenção de rotatividade dos profissionais. Em outros termos, quanto mais positiva for a percepção de práticas de responsabilidade social corporativa menor será a intenção de rotatividade. Os resultados sugerem uma interdependência entre as dimensões individual e organizacional que determinam o pensamento, o planejamento e vontade de deixar uma organização. Esta interdependência faz com que o profissional ao perceber que a sua organização adota práticas de responsabilidade social tenha um sentimento de comprometimento e confiança com a 
organização, diminuindo com isso, a intenção de rotatividade. Os dados revelaram ainda que ações de responsabilidade social voltadas para os grupos de stakeholders sociais e não sociais e empregados afetam positivamente a percepção de práticas de responsabilidade social corporativa. Os resultados levam à rejeição das hipóteses de que as ações destinadas a consumidores e governo afetam positivamente esta percepção.

Palavras-chaves: Responsabilidade Social Corporativa; Intenção de rotatividade; Modelagem de equações estruturais.

\title{
THE RELATIONSHIP BETWEEN THE PERCEPTION OF CORPORATE SOCIAL RESPONSIBILITY PRACTICES AND PROFESSIONALS' TURNOVER INTENTION.
}

\begin{abstract}
It has been analyzed in this paper the relation between the corporate social responsibility (CSR) practices perception and the turnover intention (TI) of Brazilian professionals from different states and economic sectors. The data has been collected through a questionnaire based on perceived scales of corporate social responsibility practices (TURKEY, 2009b) and turnover intention scale (Siqueira et al., 2012) with 100 professionals. The data has been analyzed by a structural equation modeling using the Smart PLS software 3. A summary of the results indicates that the perception of corporate social responsibility practices negatively influences the intention of professionals' turnover. In other words, the more positive is the corporate social responsibility practices perception implies less turnover intention. The results suggest an interdependence between the individual and organizational dimensions, which determine the thought, the planning and the desire to leave an organization. This interdependence causes the professional to realize that if his organization adopts social responsibility practices have a sense of commitment and trust towards the company, reducing then the turnover intention. The data has also revealed that social responsibility actions towards the groups of social stakeholders, not social and employees positively affect the perception of corporate social responsibility practices. The results lead to a rejection of the hypothesis that the actions aimed at consumers and government positively affect this perception.
\end{abstract}

Keywords: Corporate Social Responsibility; turnover intention; Structural equation modeling.

\section{LA RELACIÓN ENTRE LA PERCEPCIÓN DE PRÁCTICAS DE RESPONSABILIDAD SOCIAL CORPORATIVA Y LA INTENCIÓN DE ROTACIÓN DE PROFESIONALES}




\section{RESUMEN}

Se analizó en este artículo la relación entre la percepción de prácticas de responsabilidad social corporativa (RSCORP) y la intención de rotación (IR) de los profesionales brasileños de diferentes Estados y sectores económicos. Se recogieron los datos a través de un cuestionario basado en las escalas de percepción de prácticas de responsabilidad social corporativa (TURKEY, 2009b) y la escala de intención de rotación (SIQUEIRA et al, 2012) hecho a 100 profesionales. Los datos se analizaron con modelos de ecuaciones estructurales, utilizando el software Smart PLS 3. La síntesis de los resultados indica que la percepción de prácticas de responsabilidad social corporativa influye negativamente la intención de rotación de los profesionales. En otras palabras, cuanto más positiva sea la percepción de prácticas de responsabilidad social corporativa, menor será la intención de rotación. Los resultados sugieren una interdependencia entre las dimensiones individual y organizacional que determinan el pensamiento, la planificación y el deseo de dejar una organización. Esta interdependencia hace con que el profesional, al percibir que su organización adopta prácticas de responsabilidad social, tenga un sentimiento de compromiso y confianza con la organización, disminuyendo así la intención de rotación. Los datos muestran aún que las acciones de responsabilidad social dirigidas a grupos de stakeholders sociales y no sociales y empleados afectan positivamente la percepción de prácticas de responsabilidad social corporativa. Los resultados conducen al rechazo de las hipótesis de que las acciones destinadas a consumidores y gobierno afectan positivamente esta percepción.

Palabras clave: Responsabilidad Social Corporativa; Intención de Rotación; Modelo de ecuaciones estructurales.

\section{INTRODUÇÃO}

As mudanças que o ambiente de negócios tem passado nos últimos tempos vem exigindo das empresas uma maior interação com a sociedade e uma postura integradora entre as condições internas e externas, tornando cada vez mais importante o debate quanto ao grau de responsabilidade social corporativa (RSCORP) (VASCONCELOS et al., 2013). Diversos estudos que envolvem a RSCORP vêm sendo realizados, levando a confirmação de seus efeitos sobre o desempenho financeiro da empresa, na visão de consumidores e na decisão de compra, além dos efeitos na reputação empresarial, o que aporta evidências de um interesse ainda superior em relação aos impactos junto aos stakeholders externos (AGUINIS; GLAVAS, 2012). Embora alguns autores ainda apontem a existência de poucos estudos sobre os efeitos da RSCORP sobre os empregados, pesquisas já confirmam sua relação com o comprometimento organizacional, o desempenho superior, a intenção de rotatividade (IR) e os efeitos na atração de profissionais (GREENING; TURBAN, 2000; TURKER, 2009a; ALI et al., 2010; VLACHOS et al., 2010; PANAGOPOULOS et al., 2011; GAUDENCIO et al., 2014; GLAVAS; KELLEY, 2014; YILMAZ; ALI; FLOURIS, 2015). 
A retenção de profissionais nas organizações tem sido também um tema recorrente. Os fatores que contribuem para a decisão de permanência na organização têm sido estudados visando alterar as políticas de recursos humanos $(\mathrm{RH})$, permitindo uma ação preventiva e visando minimizar os riscos e custos decorrentes desta perda de capital intelectual. Torna-se, portanto, imperativo para as organizações identificar e compreender os motivos que desencadeiam a IR entre os seus empregados, de forma a promover ações que possam conter tal movimento. Estudos já desenvolvidos apontam que fatores individuais e organizacionais determinam a intenção de um indivíduo deixar uma organização de forma voluntária e que tal decisão é sustentada pela percepção que ele possui dos fatores organizacionais. A percepção dos empregados acerca de aspectos como bem-estar no trabalho, clima organizacional, estilo de liderança, suporte organizacional, comprometimento, RSCORP, comportamento ético e sua influência na IR vem sendo confirmada por diversos autores (CHANG, 1999; MELO; OLIVEIRA, 2008; YIN-FAH, FOON; CHEE-LEONG; OSMAN, 2010; POLIZZI FILHO; SIQUEIRA, 2012, LONG, 2012; KHAN, 2014).

Considerando que a IR é um comportamento do indivíduo e que este comportamento é afetado diretamente pela sua percepção, sendo ela que vai determinar a intenção de permanência refletida por meio de seu pensamento, planejamento e vontade (MOWDAY; PORTER; STEERS, 1982; SIQUEIRA et al., 2012) e considerando ainda, que as iniciativas de RSCORP podem favorecer ao desenvolvimento e manutenção do sentimento de orgulho, pertencimento e comprometimento com a organização (TURKER, 2009a; ALI et al., 2010; VLACHOS et al., 2010; PANAGOPOULOS et al., 2011; GLAVAS; KELLEY, 2014; YILMAZ; ALI; FLOURIS, 2015), definiu-se como objetivo geral deste artigo analisar a relação entre a percepção das práticas de RSCORP e a IR dos profissionais. Para isso, uma pesquisa de caráter quantitativo foi realizada com profissionais brasileiros de diferentes estados e setores econômicos e os dados foram coletados por meio de um questionário baseado nas escalas de percepção de práticas de RSCORP (TURKEY, 2009b) e escala de IR (SIQUEIRA et al., 2012).

Este estudo objetiva ainda contribuir com o aprofundamento sobre a relação da percepção de práticas de RSCORP e IR, em especial no contexto brasileiro, ainda pouco investigado cientificamente. $\mathrm{O}$ artigo está estruturado em quatro partes além da presente introdução. $\mathrm{Na}$ segunda, apresenta-se o referencial teórico, na terceira, os aspectos metodológicos e, na sequência, a apresentação e discussão dos resultados e as conclusões. 


\section{CONSIDERAÇÕES SOBRE A RESPONSABILIDADE SOCIAL CORPORATIVA E A INTENÇÃO DE ROTATIVIDADE}

Ao se discutir RSCORP duas linhas distintas são encontradas: a tradicional, que considera que a empresa ao focar no mercado e em sua responsabilidade de gerar lucro cumpre com seu papel (FRIEDMAN, 1970) e uma segunda, que defende que as organizações devam ter uma ação que vai além das questões econômicas, englobando outras dimensões (CAROLL, 1991, 1999; TURKER, 2009a). As profundas mudanças que o ambiente de negócios tem passado nos últimos tempos vem exigindo das empresas uma maior interação com a sociedade e uma postura integradora entre as condições internas e externas, tornando o debate quanto ao grau de responsabilidade social das organizações cada vez mais importante (VASCONCELOS et al., 2013)

O argumento de que no conceito de RSCORP estão contempladas as expectativas éticas, legais, econômicas e discricionárias que a sociedade tem perante as organizações em determinado momento é apresentado por Carroll (1991), que propõe um modelo de pirâmide de responsabilidade social corporativa, onde considera que a responsabilidade econômica é base para os demais níveis de responsabilidade (legal, ética e discricionária) que devem ser cumpridas de forma simultânea. Para o autor, uma organização socialmente responsável é aquela cujo modelo de gestão se esforça para gerar lucros, cumprir com as obrigações legais, agir com ética e ter uma boa cidadania corporativa com diferentes grupos de interesse.

É na década de 90 que se observa que o conceito de RSCORP ganha maturidade e se consolida no entendimento de que é uma prática de gestão empresarial que, preocupada com os interesses de seus stakeholders, busca atender às expectativas de ordem econômica, social e ambiental. Assim, ao atender tais expectativas em produtos e serviços, empresas são consideradas como aquelas que atuam de forma ética e visam alcançar sucesso em seus negócios, incluindo uma boa reputação (HART; MILSTEIN, 2004; HERRMANN, 2004; PORTER; KRAMER; 2002, 2006; MOON, 2007). Com esta visão, a RSCORP passa a ser considerada como função do negócio, recebendo tratamento com o mesmo status da qualidade, da marca, dos produtos e normatizando padrões de relacionamentos entre diferentes atores sociais.

Nos estudos que defendem a vertente estratégica da RSCORP (HART; MILSTEIN, 2004; HERRMANN, 2004; PORTER; KRAMER, 2002, 2006; MOON, 2007), encontram-se os argumentos de que ela deve ser considerada uma vantagem competitiva e de geração de valor para todas as partes interessadas. Assim, as ações de RSCORP, quando atreladas aos 
propósitos estratégicos das organizações, criam melhoria na reputação e imagem, possibilitam maior motivação e, consequente, retenção de pessoas. Com isso, viabilizam a criação de projetos inovadores e eficientes que possibilitam um melhor desempenho econômico, ao mesmo tempo que mantém o alinhamento com estratégias de desempenho ambiental e social, assim como a melhoria na governança corporativa. Embora esta linha seja a mais hegemônica, percebe-se um interesse e crescimento de uma variante sistêmica onde os negócios empresariais passam a considerar as expectativas dos stakeholders em seus processos organizacionais (FREEMAN, 1984). Ao identificar os papéis de cada grupo, a empresa busca entender como cada um deles interfere em sua reputação, na sua sobrevivência, e estabelece as bases para desenvolver e manter a confiança na organização (TURKER, 2009a; ALI et al., 2010; VLACHOS et al., 2010).

Turker (2009a) defende que a RSCORP se refere a comportamentos corporativos que afetam stakeholders positivamente e que vão além dos interesses econômicos da empresa. O autor argumenta ainda, que as ações de RSCORP podem ser classificadas em quatro tipos, considerando grupos de interesse distintos: (1) stakeholders sociais e não sociais (RSA) englobando ações voltadas para a sociedade, meio ambiente, gerações futuras e organizações não-governamentais. É um grupo formado por partes interessadas no negócio que não afetam ou são afetadas diretamente pela organização, apesar de se apresentarem muito importantes; (2) os empregados (RSE): com foco em ações voltadas para as condições de trabalho incluindo justiça, carreira, educação, remuneração, decisões justas, equilíbrio entre vida pessoal e profissional, bem como incentivo a práticas de voluntariado. Este é o grupo de grande importância pois a percepção das ações desenvolvidas além de afetar diretamente o empregado, seu resultado potencialmente gerará maior comprometimento e consequentemente maior disposição para pôr em prática as demais ações de responsabilidade social da empresa; (3) os consumidores (RSC): com ações que buscam a proteção dos direitos além dos legais, o fornecimento de informações sobre produtos e serviços, e a consequente satisfação do consumidor; e (4) o governo (RSG): com ações que identifiquem o cumprimento dos aspectos legais de forma completa e imediata.

Para Turker (2009a), quando uma empresa apresenta práticas nestes quatro níveis e, que são percebidas pelos empregados de forma positiva, a tendência é que gere maior comprometimento e orgulho, já que ninguém quer trabalhar em uma empresa que não trata bem seus clientes, não respeita as leis, não cuida do seu entorno, das gerações futuras e nem de seus empregados. O modelo proposto por Turker (2009a) está em consonância com a 
proposição de Caroll (1991), que é ainda nos dias atuais o modelo mais amplamente aceito. Entretanto, cabe destacar que a dimensão da responsabilidade social voltada para o governo, proposta por Turker (2009a), embora esteja em consonância com o modelo de Caroll (1991) reafirma a dimensão legal da responsabilidade social, fator que ainda apresenta pontos de vistas divergentes na literatura. Para fins de operacionalização deste estudo, adota-se o conceito e o modelo de RSCORP propostos por Turker (2009b).

Diversos estudos que envolvem a RSCORP junto aos stakeholders externos vêm sendo realizados e confirmam os efeitos sobre o desempenho financeiro da empresa (ADEWALE; RAHMON, 2014), na decisão de compra (SEN; BHATTACHARYA, 2001) e na decisão de fornecimento (YOUNG; MAKHIJA, 2014), evidenciando um interesse ainda superior em relação aos impactos da RSCORP junto aos stakeholders externos (AGUINIS; GLAVAS, 2012). Por outro lado, observa-se também que estudos mais recentes vêm sendo conduzidos sobre a percepção dos empregados e os efeitos desta percepção sobre as atitudes e comportamentos organizacionais. Estes estudos apontam que quando uma empresa tem iniciativas de RSCORP, os empregados tendem a se sentirem mais orgulhosos e comprometidos com a organização (TURKER, 2009a; ALI et al., 2010; VLACHOS et al., 2010; PANAGOPOULOS et al., 2011; GLAVAS; KELLEY, 2014; YILMAZ; ALI; FLOURIS, 2015), refletindo em comportamentos de cidadania organizacional (ELLIS, 2009), desempenho superior (GAUDENCIO et al., 2014) e atração de novos empregados (GREENING; TURBAN, 2000).

Nesta pesquisa há um interesse em analisar a influência da RSCORP na IR. Isso porque a retenção de profissionais, apesar de pouco explorado na literatura, tem sido um tema recorrente nas organizações em função do impacto que a perda de profissionais traz para os projetos estratégicos e a competitividade (GLAVAS; KELLEY, 2014; KHAN, 2014). Ao decidirem deixar de forma voluntária uma organização, os empregados podem provocar repercussões negativas para as empresas, representando custos tangíveis e intangíveis tais como: perda de conhecimento, quebra de vínculos com stakeholders externos, mudanças e/ou interrupções no fluxo de trabalho (SIQUEIRA et al., 2012).

A IR é considerada como um fenômeno de natureza psicológica que se refere a probabilidade estimada de um indivíduo deixar uma organização em algum momento, expressa por meio de seu pensamento, planejamento ou da vontade (MOWDAY; PORTER; STEERS, 1982; SIQUEIRA et al., 2012). Neste sentido, cada vez mais as organizações tentam descobrir as 
razões que desencadeiam a IR entre os seus empregados e buscam atuar preventivamente por meio de suas práticas de RH, assegurando a retenção (SCOTT, 2012).

Os fatores que determinam a IR podem ser agrupados em fatores individuais e fatores organizacionais. Nos fatores individuais, aponta-se a personalidade do indivíduo e a sua influência na percepção como fatores que permitem que os profissionais tenham acesso a informações, interpretem, classifiquem e reajam de forma diferente aos diversos estímulos. Dentre os fatores organizacionais apontados, destaca-se a qualidade do ambiente de trabalho e a relação direta com o grau de estresse e de conflitos, associado a oportunidades de crescimento e de desenvolvimento (JHA, 2009; HONGVICHIT, 2015).

De modo a identificar a relação entre os fatores individuais e organizacionais, a literatura aponta a existência de diversos estudos que buscam analisar a influência da percepção dos empregados em relação ao bem-estar no trabalho, clima organizacional, estilo de liderança, suporte organizacional, comprometimento, RSCORP, comportamento ético e seu impacto na IR e, como resultado, estes estudos encontraram evidências que a percepção positiva sobre tais aspectos reduzem a probabilidade de um profissional deixar a organização de forma voluntária (CHANG, 1999; MELO;OLIVEIRA, 2008; YIN-FAH, FOON; CHEE-LEONG; OSMAN, 2010; POLIZZI FILHO; SIQUEIRA, 2012, LONG, 2012; KHAN, 2014; YOO et al., 2015).

Adicionalmente, outros estudos que relacionam a percepção dos empregados sobre práticas de RSCORP com a IR têm em comum a constatação que empresas, quando desenvolvem ações de RSCORP que são percebidas de forma positiva pelos empregados, tendem a ver redução na IR (HELM, 2013; KHAN, 2014; GAUDENCIO et al., 2014; YILMAZ; FLOURIS, 2015; YOO et al., 2015).

Neste artigo, entende-se que a IR é um comportamento do indivíduo que se refere à probabilidade estimada de um indivíduo deixar uma organização em algum momento, expressa por meio de seu pensamento, planejamento e vontade e que este comportamento é afetado diretamente pela sua percepção (MOWDAY; PORTER; STEERS, 1982; SIQUEIRA et al., 2012). Considera-se ainda, que as iniciativas de RSCORP podem favorecer ao desenvolvimento e manutenção do sentimento de orgulho, pertencimento e comprometimento com a organização (TURKER, 2009a; ALI et al., 2010; VLACHOS et al., 2010; PANAGOPOULOS et al., 2011; GLAVAS; KELLEY, 2014; YILMAZ; ALI; FLOURIS, 2015), contribuindo assim para o desejo de permanência de mais longo prazo e consequente redução na intenção de deixar a organização. Com base em tal argumentação propõe-se 
para este estudo as seguintes hipóteses de pesquisa representadas pelo modelo nomotético, conforme Figura 1.

Hipótese 1 - A percepção positiva de práticas de responsabilidade social corporativa (RSCORP) influencia negativamente na intenção de rotatividade (IR).

Hipótese 2 - As ações de responsabilidade social voltadas para os stakeholders sociais e não sociais (RSA) afetam positivamente a percepção de responsabilidade social corporativa (RSCORP).

Hipótese 3 - As ações de responsabilidade social voltadas para os empregados (RSE) afetam positivamente a percepção de responsabilidade social corporativa (RSCORP).

Hipótese 4 - As ações de responsabilidade social voltadas para os consumidores (RSC) afetam positivamente a percepção de responsabilidade social corporativa (RSCORP).

Hipótese 5 - As ações de responsabilidade social voltadas para o governo (RSG) afetam positivamente a percepção de responsabilidade social corporativa (RSCORP).

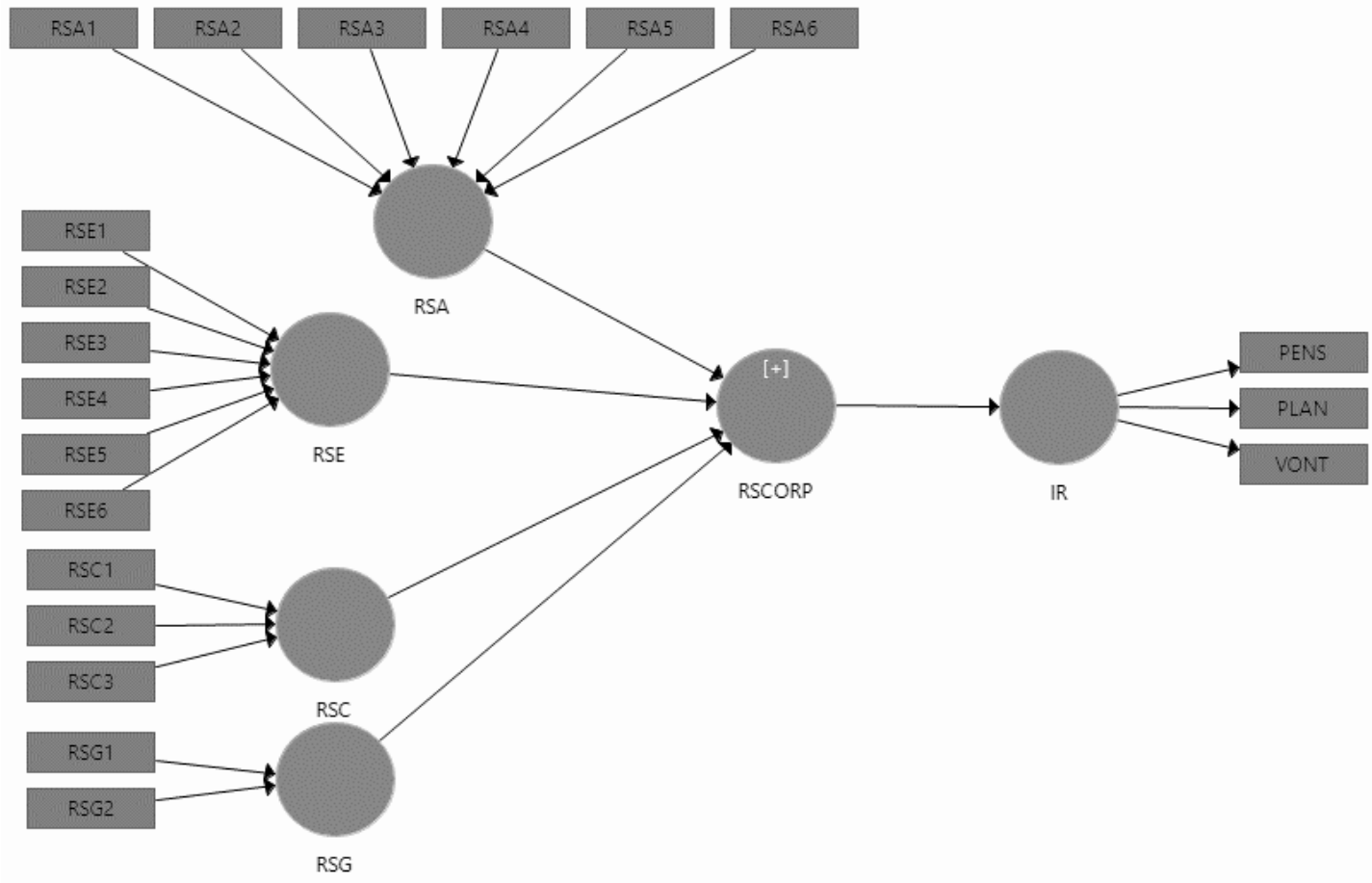

Figura 1 - Modelo nomotético da pesquisa

Fonte: Desenvolvido pelos autores

\section{METODOLOGIA}

A população alvo do estudo é caracterizada por profissionais de nível técnico e superior de diferentes Estados e setores econômicos que possuíam cadastro em um banco de dados com 
abrangência nacional de uma consultoria de RH. A coleta foi realizada entre os meses de setembro e outubro de 2015 e se deu por meio de um instrumento dividido em blocos, sendo a $1^{\mathrm{a}}$ parte com dados demográficos, a segunda e a terceira baseado nas escalas de percepção de práticas RSCORP (TURKEY, 2009b) e intenção de rotatividade - EIR (SIQUEIRA et al., 2012), respectivamente. O questionário foi distribuído para 2000 profissionais utilizando-se o formulário de pesquisa online Google apps for work, obtendo-se o retorno de 100 questionários, representando $5 \%$ de taxa de retorno. O tamanho da amostra foi considerado suficiente para o algoritmo do PLS por atender ao requisito mínimo de dez vezes o número de indicadores do construto com maior número de indicadores (HAIR JR et al., 2014).

A escala de percepção de práticas de RSCORP foi desenvolvida por Turker (2009b) e mede a percepção do profissional sobre a responsabilidade social de uma organização. Esta escala possui 17 itens, separados em quatro tipos de grupos de interesse para os quais as ações de RSCORP são voltadas, sendo: seis assertivas para o grupo stakeholders sociais e não-sociais (RSA), seis para o grupo empregados (RSE), três para o grupo consumidores (RSC) e dois para o grupo governo (RSG). As assertivas foram avaliadas em uma escala Likert de cinco pontos, indo de (1) discordo totalmente até (5) concordo totalmente, conforme Quadro 1 apresentado a seguir.

\section{Quadro 1 - Escala de percepção de práticas de responsabilidade social corporativa} (RSCORP)

\begin{tabular}{|c|c|}
\hline Variável & Questão \\
\hline \multirow{6}{*}{$\begin{array}{l}\text { RSA } \\
\text { Responsabilidade } \\
\text { social voltada para } \\
\text { stakeholders social e } \\
\text { não social }\end{array}$} & $\begin{array}{l}\text { RSA1 - Minha empresa incentiva atividades, internas ou externas, } \\
\text { que visam proteger e melhorar a qualidade do meio ambiente. }\end{array}$ \\
\hline & $\begin{array}{l}\text { RSA2 - Minha empresa investe na criação de uma vida melhor } \\
\text { para gerações futuras. }\end{array}$ \\
\hline & $\begin{array}{l}\text { RSA3 - Minha empresa implementa programas especiais para } \\
\text { minimizar os seus impactos negativos no meio ambiente. }\end{array}$ \\
\hline & $\begin{array}{l}\text { RSA4 - Minha empresa busca um crescimento sustentável, } \\
\text { considerando gerações futuras. }\end{array}$ \\
\hline & $\begin{array}{l}\text { RSA5 - Minha empresa atua, mesmo que indiretamente, em áreas } \\
\text { problemáticas. }\end{array}$ \\
\hline & $\begin{array}{l}\text { RSA6 - Minha empresa contribui com campanhas e projetos que } \\
\text { promovem o bem-estar da sociedade. }\end{array}$ \\
\hline RSE & RSE 1 - Minha empresa incentiva seus empregados a participar de \\
\hline
\end{tabular}




\begin{tabular}{|c|c|}
\hline \multirow{6}{*}{$\begin{array}{l}\text { Responsabilidade } \\
\text { social voltada para } \\
\text { empregados }\end{array}$} & atividades voluntárias. \\
\hline & $\begin{array}{l}\text { RSE } 2 \text { - As políticas da minha empresa incentivam os empregados } \\
\text { a desenvolver suas competências e carreiras. }\end{array}$ \\
\hline & $\begin{array}{l}\text { RSE } 3 \text { - A gestão da minha empresa se preocupa com as } \\
\text { necessidades e desejos dos empregados. }\end{array}$ \\
\hline & $\begin{array}{l}\text { RSE } 4 \text {-Minha empresa implementa políticas que possibilitam um } \\
\text { bom equilíbrio entre trabalho e vida pessoal aos empregados }\end{array}$ \\
\hline & $\begin{array}{l}\text { RSE } 5 \text { - As decisões gerenciais relacionadas com os empregados } \\
\text { são, de maneira geral, justas. }\end{array}$ \\
\hline & $\begin{array}{l}\text { RSE } 6 \text { - Minha empresa investe em treinamento sugeridos por } \\
\text { seus empregados. }\end{array}$ \\
\hline \multirow{3}{*}{$\begin{array}{l}\text { RSC } \\
\text { Responsabilidade } \\
\text { social voltada para } \\
\text { consumidores }\end{array}$} & $\begin{array}{l}\text { RSC } 1 \text { - Minha empresa protege os direitos dos consumidores } \\
\text { além das exigências legais. }\end{array}$ \\
\hline & $\begin{array}{l}\text { RSC } 2 \text { - Minha empresa fornece informações completas e precisas } \\
\text { sobre seus produtos aos seus consumidores. }\end{array}$ \\
\hline & $\begin{array}{l}\text { RSC } 3 \text { - O foco na satisfação dos clientes é muito importante para } \\
\text { a minha empresa. }\end{array}$ \\
\hline \multirow{2}{*}{$\begin{array}{l}\text { RSG } \\
\text { Responsabilidade } \\
\text { social voltada para } \\
\text { governos }\end{array}$} & $\begin{array}{l}\text { RSG } 1 \text { - Minha empresa sempre paga seus impostos de maneira } \\
\text { regular e contínua. }\end{array}$ \\
\hline & $\begin{array}{l}\text { RSG } 2 \text { - Minha empresa cumpre os regulamentos legais de forma } \\
\text { completa e imediata. }\end{array}$ \\
\hline
\end{tabular}

Fonte: Turker (2009b).

A escala de intenção de rotatividade (EIR) foi desenvolvida e validada por Siqueira et al. (2012) e já utilizada em diversos estudos nacionais. A EIR avalia o grau em que um indivíduo elabora planos sobre sua saída da empresa em que trabalha. É composta por 3 assertivas que permitem que os indivíduos declarem o que pensa, planeja e tem vontade acerca da possiblidade de deixar sua organização no futuro, avaliadas em uma escala Likert de cinco pontos, indo de (1) nunca até (5) sempre, que são apresentadas no Quadro 2 a seguir.

Quadro 2 - Escala de intenção de rotatividade (EIR)

\begin{tabular}{|l|l|}
\hline Variável & Questão \\
\hline Planejamento & Planejo sair da empresa onde trabalho \\
\hline
\end{tabular}




\begin{tabular}{|l|l|}
\hline Pensamento & Penso em sair da empresa onde trabalho \\
\hline Vontade & Tenho vontade de sair da empresa onde trabalho \\
\hline
\end{tabular}

Fonte: - Siqueira et al. (2012)

Para estimar a relação entre a percepção de práticas de RSCORP e a IR dos profissionais utilizou-se a modelagem de equações estruturais com o software Smart PLS 3 (RINGLE; WENDE; BECKER, 2014). Esta opção se justifica por possibilitar a análise de múltiplas relações simultaneamente, por permitir a modelagem da relação entre conceitos não observados diretamente, por permitir a análise de modelos formativos e pelo tamanho de amostra requerida (HAIR JR et al., 2014; RINGLE; WENDE; BECKER, 2014)

Para proceder com a análise, observou-se os estágios na modelagem de equações estruturais, conforme proposto por HAIR JR et al. (2014). Após as especificações dos modelos estrutural e de mensuração, procedeu-se com a (1) coleta e exame dos dados; (2) a estimação dos coeficientes de caminho; (3) a avaliação dos modelos de mensuração reflexivo e formativo e; (4) a avaliação do modelo estrutural.

Nesta pesquisa não foram identificados dados ausentes, não sendo, portanto, necessária a utilização de técnicas como a substituição de dados ausentes pela média, ou exclusões pairwise ou listwise nos dados dos 100 questionários devolvidos pelos respondentes (HAIR JR et al., 2014).

\section{APRESENTAÇÃO E DISCUSSÃO DOS RESULTADOS}

\section{1 -Caracterização da amostra}

Os dados demográficos indicam que 59\% dos respondentes são do sexo feminino, $43 \%$ tem até 40 anos de idade, $62 \%$ possuem MBA, $63 \%$ ocupam posição de liderança e $49 \%$ estão na empresa até 6 anos, sendo que $61 \%$ atuam no setor de serviços, $27 \%$ em indústrias e $12 \%$ em comércio. Os respondentes residem nas regiões sul e sudeste, com a seguinte distribuição: Espírito Santo (79\%), seguido dos estados do Rio de Janeiro (9\%), São Paulo (5\%), Paraná (4\%) e Minas Gerais (3\%).

\subsection{Avaliação do modelo de mensuração}

Foram avaliadas a consistência interna, a confiabilidade do indicador, a validade convergente e a validade discriminante utilizando-se os critérios de FornelLarcker e Cross Loading. A confiabilidade da consistência interna das escalas foi avaliada usando-se o coeficiente Alpha 
de Cronbach, que é baseado no número de itens em uma escala e na intercorrelação entre os itens, ou seja, quanto maior for a inter-relação entre os itens, maior é a confiabilidade de toda a escala (HAYES, 2001). Os resultados desta avaliação indicaram confiabilidade composta de 0,969 e um Alpha de Cronbach de 0,952, estando as duas medidas dentro dos valores aceitáveis, conforme recomendações de Hair Jr et al. (2014). Posteriormente, avaliou-se a confiabilidade do indicador e todos apresentaram outerloadings acima de 0,708, indicando alta associação. Na avaliação da validade convergente, extensão que indica a correlação positiva com medidas do mesmo construto, encontrou-se uma AVE de 0,913, indicando que o modelo converge a um resultado satisfatório por apresentar resultados maiores que 0,50 (HAIR JR et al., 2014). Por fim, avaliou-se a validade discriminante, que é à medida que avalia o grau de independência dos indicadores ou variáveis latentes em relação a outros itens, utilizando-se os critérios de FornelLarcker e Cross Loading e para os dois testes a validade discriminante foi assegurada (RINGLE et al., 2014). A Tabela 1 a seguir demonstra o resumo da avaliação do modelo de mensuração reflexivo.

Tabela 1 - Modelo de mensuração reflexivo

\begin{tabular}{|c|c|c|c|c|c|c|}
\hline $\begin{array}{l}\text { Variável } \\
\text { latente }\end{array}$ & Indicador & $\begin{array}{l}\text { Confiabilida } \\
\text { de composta }\end{array}$ & $\begin{array}{c}\text { Alpha de } \\
\text { Cronbac } \\
\text { h }\end{array}$ & $\begin{array}{c}\text { Confiabilida } \\
\text { de do } \\
\text { indicador }\end{array}$ & $\begin{array}{c}\mathbf{A V} \\
\mathbf{E}\end{array}$ & $\begin{array}{c}\text { Validade } \\
\text { discriminan } \\
\text { te }\end{array}$ \\
\hline \multirow{3}{*}{$\begin{array}{l}\text { Intenção } \\
\text { de } \\
\text { rotativida } \\
\text { de }\end{array}$} & Pensamento & 0,969 & 0,952 & 0,960 & \multirow{3}{*}{$\begin{array}{c}0,91 \\
3\end{array}$} & Sim \\
\hline & $\begin{array}{c}\text { Planejamen } \\
\text { to }\end{array}$ & & & 0,943 & & Sim \\
\hline & Vontade & & & 0,964 & & Sim \\
\hline
\end{tabular}

Fonte: Dados da pesquisa

Na sequência, avaliou-se a multicolinearidade e a significância e relevância dos indicadores formativos de $1^{\mathrm{a}}$ ordem (RSA, RSE, RSC e RSG). Embora haja a indicação para avaliar a validade convergente (HAIR JR et al., 2014), esta avaliação não foi realizada em função dos construtos formativos deste modelo não apresentarem variáveis reflexivas. A não proposição de variáveis reflexivas se deu pela decisão de manter preservada a escala de Turker (2009b) em função da adequação e da utilização em outros estudos e por ser suficiente ao que se propunha a pesquisa, optando-se por um questionário mais parcimonioso.

Posteriormente avaliou-se a multicolinearidade por meio do VIF (variance inflation factor), que mede o grau em que a variância dos coeficientes é afetada pela multicolinearidade, cujos parâmetros são: VIF = 1 não correlacionado, entre 1 e 5 moderadamente correlacionado e 
acima de 5 altamente correlacionado (HAIR JR et al., 2014). Observou-se que quase todos os indicadores tiveram correlações moderadas, à exceção dos indicadores RSA4 e RSE 3 que apresentaram alta correlação, conforme Tabela 2. Considerando que os dois indicadores contribuem junto com os demais para o entendimento do construto, conforme aponta a literatura, os indicadores foram mantidos no modelo para preservar a definição do construto já que ao retirá-lo corre-se o risco de alterar o construto (BIDO et al., 2009; HAIR JR et al., 2014).

Tabela 2 - VIF - variáveis de $2^{\mathrm{a}}$ ordem (variance inflation factor)

\begin{tabular}{cccc}
\hline Variável & VIF & Variável & VIF \\
\hline RSA1 & 3,045 & RSE1 & 2,526 \\
\hline RSA2 & 3,705 & RSE2 & 3,161 \\
\hline RSA3 & 2,825 & RSE3 & 5,518 \\
\hline RSA4 & 5,435 & RSE4 & 3,864 \\
\hline RSA5 & 1,528 & RSE5 & 2,834 \\
\hline RSA6 & 3,015 & RSE6 & 2,543 \\
\hline RSC1 & 3,356 & RSG1 & 2,690 \\
\hline RSC2 & 3,621 & RSG2 \\
\hline RSC3 & 3,129 & 4,378 \\
\hline \multicolumn{4}{c}{ Fonte: Dados da pesquisa }
\end{tabular}

A avaliação da significância e relevância dos indicadores formativos por meio do teste $\mathrm{T}$ gerado pelo procedimento do bootstrapping, indicou que somente os indicadores formativos (RSA4, RSC1, RSE3, RSE5, RSG2) estabeleceram valores T dentro dos níveis especificados para a aceitação (0,363). Entretanto, ao analisar os valores de crossloadings (cargas), identificou-se que todos possuem carga acima de 0,50 , demonstrando a relevância teórica para o modelo, e por este motivo optou-se por mantê-los (BIDO et al., 2009).

Para mensurar os indicadores formativos de $2^{\mathrm{a}}$ ordem, calculou-se os escores das variáveis latentes dos indicadores formativos de $1^{\text {a }}$ ordem e um novo modelo foi apresentado, conforme 
Figura 2, reiniciando a avaliação do modelo de mensuração formativo.

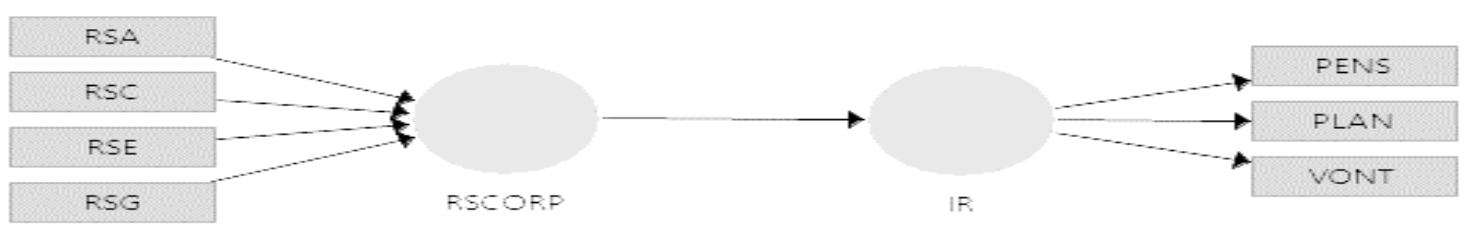

\section{Figura 2 - Modelo teórico da pesquisa}

Fonte: Desenvolvido pelos autores

Avaliando-se a multicolinearidade dos indicadores de $1^{\mathrm{a}}$ ordem observou-se que apresentaram correlações moderadas, conforme resultados que seguem: RSA (VIF =1,017), RSC (VIF $=1,090)$, RSE (VIF=1,135) e RSG (VIF=1,198) VIF. Este resultado corrobora que a medida proposta por Turker (2009b) é capaz de capturar a percepção das práticas de RSCORP.

$\mathrm{Na}$ avaliação dos outerloadings e outerweights, por meio do teste $\mathrm{T}$ gerado pelo procedimento do bootstrapping, indicou que os indicadores formativos (RSC) e (RSG) estabeleceram valores $\mathrm{T}$ acima dos valores especificados para a aceitação, conforme demonstra a Tabela 3, optando-se pela exclusão do indicador RSG e a manutenção do indicador RSC. Esta decisão se deu pelo fato de que RSC, embora apresente carga menor que 0,50 possui sustentação teórica para sua manutenção. Argumenta-se que as medidas de RSG podem estar refletindo as discussões existentes na literatura de que atuar de forma legal, com pagamento de impostos e obediência às leis, não seja um indicativo de responsabilidade social e sim, um comportamento mais do que esperado para qualquer organização. Argumenta-se ainda, que pode ter sido favorecido pela forma de escrita do indicador, que trata não das ações que extrapolam a legalidade, tal como proposto por Caroll (1991), mas sim, por agir de forma imediata e dentro dos padrões legais, o que argumenta Turkey (2009b). Os valores apresentados demonstram ainda, que os indicadores RSE e RSA contribuem de forma mais significativa para a construção do construto RSCORP. Este resultado corrobora os estudos de Turkey (2009b) que aponta que esses grupos de stakeholders são afetados ou afetam diretamente a organização e que práticas destinadas a eles ampliam a percepção de responsabilidade social de uma organização. 
Tabela 3 - Resultados de outerloadings e outerweight

\begin{tabular}{llllll}
\hline Outerweight & & & & & \\
\hline & $\begin{array}{l}\text { Original } \\
\text { Sample } \\
(\mathbf{O})\end{array}$ & $\begin{array}{l}\text { Sample } \\
\text { Mean } \\
(\mathbf{M})\end{array}$ & $\begin{array}{l}\text { Standard } \\
\text { Deviation } \\
\text { (STDEV) }\end{array}$ & $\begin{array}{l}\text { T Statistics } \\
(\mid \mathbf{O} \text { STDEV|) }\end{array}$ & $\begin{array}{l}\text { P } \\
\text { Values }\end{array}$ \\
\hline $\begin{array}{l}\text { RSA -> } \\
\text { RSCORP }\end{array}$ & 0,539 & 0,513 & 0,182 & 2,958 & 0,003 \\
\hline $\begin{array}{l}\text { RSC -> } \\
\text { RSCORP }\end{array}$ & 0,234 & 0,228 & 0,192 & 1,220 & 0,223 \\
\hline $\begin{array}{l}\text { RSE -> } \\
\text { RSCORP }\end{array}$ & 0,693 & 0,643 & 0,181 & 3,821 & 0,000 \\
\hline $\begin{array}{l}\text { RSG -> } \\
\text { RSCORP }\end{array}$ & 0,086 & 0,101 & 0,224 & 0,386 & 0,699 \\
\hline
\end{tabular}

Outerloadings

\begin{tabular}{llllll}
\hline & $\begin{array}{l}\text { Original } \\
\text { Sample } \\
(\mathbf{O})\end{array}$ & $\begin{array}{l}\text { Sample } \\
\text { Mean } \\
(\mathbf{M})\end{array}$ & $\begin{array}{l}\text { Standard } \\
\text { Deviation } \\
(\text { STDEV })\end{array}$ & $\begin{array}{l}\text { T Statistics } \\
(\mid \mathbf{O} / \text { STEV })\end{array}$ & $\begin{array}{l}\text { P } \\
\text { Values }\end{array}$ \\
\hline $\begin{array}{l}\text { RSA -> } \\
\text { RSCORP }\end{array}$ & 0,610 & 0,572 & 0,166 & 3,674 & 0,000 \\
\hline $\begin{array}{l}\text { RSC -> } \\
\text { RSCORP }\end{array}$ & 0,407 & 0,378 & 0,188 & 2,162 & 0,031 \\
\hline $\begin{array}{l}\text { RSE - } \\
\text { RSCORP }\end{array}$ & 0,782 & 0,728 & 0,139 & 5,625 & 0,000 \\
\hline $\begin{array}{l}\text { RSG -> } \\
\text { RSCORP }\end{array}$ & 0,395 & 0,377 & 0,215 & 1,841 & 0,066 \\
\hline
\end{tabular}

Fonte: Dados da pesquisa

Desta forma, prosseguiu-se com avaliação do modelo estrutural na medida em que todos os demais indicadores formativos de $1^{\mathrm{a}}$ e $2^{\mathrm{a}}$ ordem e os reflexivos apresentam níveis aceitáveis de qualidade.

\subsection{Avaliação do modelo de estrutural}

Esta avaliação permite testar se os dados da pesquisa dão suporte ao modelo teórico proposto e para isso seguiu-se com as recomendações quanto as etapas a serem cumpridas de avaliação (1) da multicolinearidade do modelo estrutural; (2) da relevância e significância do modelo estrutural; (3) da avaliação do nível de $\mathrm{R}^{2}$ e; (4) da relevância preditiva $\mathrm{Q}^{2}$ e os efeitos do tamanho de q2 (HAIR JR et al., 2014).

$\mathrm{Na}$ avaliação da multicolinearidade, obtida por meio do VIF (variance inflation factor), constatou-se que não há presença de colinearidade já que todos os valores de VIF estavam nos 
níveis aceitáveis (HAIR JR et al., 2014), (RSE e RSA - VIF = 1,017). Na sequência, a avaliação da significância e relevância do modelo estrutural foi realizada por meio do teste $\mathrm{T}$ gerado pelo procedimento do bootstrapping. Os estudos apontam que práticas de responsabilidade social destinada aos clientes impactam positivamente na percepção de RSCORP. Exceto o RSC, todas as demais relações (RSE e RSA) e as relações com IR foram confirmadas e a maior significância se encontra em RSE, confirmando que quando empregados são afetados por práticas que considerem justas há um aumento de percepção de RSCORP. Os resultados obtidos indicam que a relação RSC com RSCORP ficou abaixo dos valores de referência $(1,65 ; 1,96 ; 2,57)$. Tal comportamento pode estar associado a uma visão diferenciada do consumidor brasileiro, que a exemplo de estudo conduzido por Maignan (2001), apontou que em alguns países, as preocupações se as empresas estão em conformidade com padrões legais e éticos geram maior percepção da responsabilidade social. O público pesquisado pode não apresentar esta preocupação, o que pode ser explorado em pesquisas futuras.

Prosseguiu-se com a avaliação do nível de $\mathrm{R}^{2}$ que indicou um valor de 38,6\% da RSCORP sobre a IR. Esta medida explica a porção da variação da variável endógena (IR) a partir da variável exógena (RSCORP) e os valores encontrados apontam para um efeito grande, conforme Ringle et al., 2014. Este resultado confirma pesquisas já existentes que demonstram que quando as empresas desenvolvem ações de RSCORP que são percebidas de forma positiva pelos empregados, tendem a ver redução na IR (HELM, 2013; KHAN, 2014; GAUDENCIO et al., 2014; YILMAZ; FLOURIS, 2015; YOO et al., 2015).

Por fim, avaliou-se a qualidade da predição do modelo. Nesta medida devem ser obtidos valores maiores que zero, sabendo-se que um modelo perfeito teria um Q2 $=1$ (HAIR JR et al., 2014). O resultado é obtido por meio do Blindfolding, calculando-se o cross-validated redundancy, que engloba estimativas do modelo estrutural e de mensuração. Os valores encontrados foram maiores do que zero $(0,112)$, indicando que os construtos têm relevância preditiva para o modelo.

A partir das validações obtidas com o modelo de mensuração e estrutural, apresenta-se no Quadro 3 a síntese dos resultados dos testes de hipóteses do estudo.

Quadro 3 - Teste de hipóteses da pesquisa

Hipótese

Resultado

Hipótese 1 - A percepção de práticas de responsabilidade social Não rejeitada corporativa (RSCORP) influencia negativamente na intenção de rotatividade (IR). 
Hipótese 2 - As ações de responsabilidade social voltadas para os Não rejeitada stakeholders sociais e não sociais (RSA) afetam positivamente a percepção de responsabilidade social corporativa (RSCORP).

Hipótese 3 - As ações de responsabilidade social voltadas para os Não rejeitada empregados (RSE) afetam positivamente a percepção de responsabilidade social corporativa (RSCORP).

Hipótese 4 - As ações de responsabilidade social voltadas para os Rejeitada consumidores (RSC) afetam positivamente a percepção de responsabilidade social corporativa (RSCORP).

Hipótese 5 - As ações de responsabilidade social voltadas para o governo Rejeitada (RSG) afetam positivamente a percepção de responsabilidade social corporativa (RSCORP).

Fonte: Dados da pesquisa

Das cinco hipóteses testadas constatou-se que as três primeiras não foram rejeitadas e as duas últimas foram rejeitadas. O teste da primeira hipótese aponta evidências de que a percepção de práticas de RSCORP influencia negativamente a IR. Colocado de outra forma, os resultados indicam que quanto mais positiva for a percepção de práticas de RSCORP menor será a IR por parte dos profissionais participantes da pesquisa. Esses resultados sugerem uma relação de interdependência entre os fatores individuais e organizacionais que determinam a IR (JHA, 2009; HONGVICHIT, 2015). Em parte, esta interdependência demonstra que a organização que adota práticas de RSCORP influencia dois antecedentes da IR, quais sejam, o comprometimento e a confiança (CARMO, 2009).

Costa e Bastos (2014) e Bastos (1993) apontam a relevância do campo de pesquisa sobre o comprometimento organizacional e sobre os vínculos entre o indivíduo e a sua organização empregadora. Nesse contexto, Bastos (1993) identifica a existência de estudos sobre os antecedentes, os correlatos e os consequentes de comprometimento organizacional e enfatiza que uma das principais classes de comportamento que tem sido analisada como consequência do comprometimento organizacional é a IR. Para Bastos (1993) as pesquisas neste domínio apoiam-se no postulado de que o alto comprometimento leva a menor rotatividade, baixo absenteísmo e melhoria de desempenho.

Já a confiança do profissional na organização pode ser definida como as crenças a respeito de padrões éticos, credibilidade da comunicação, poder econômico da organização e capacidade desta de reconhecer o desempenho do empregado, tanto financeira quanto profissionalmente (OLIVEIRA, 2004). No caso em questão, a perspectiva de confiança como antecedente da IR está relacionado aos padrões éticos e credibilidade conquistada pela organização com a prática de RSCORP junto a seus stakeholders e a sociedade. 
Morgan e Hunt (1994) afirmam que o desenvolvimento de comprometimento e de confiança ocorre quando as organizações sustentam suas relações, entre outros aspectos, (a) por meio do fornecimento de recursos, de oportunidades e de benefícios que são maiores que outras possibilidades de parcerias; (b) que mantém elevados valores de padrões corporativos alinhando-os a outros parceiros com valores semelhantes para compartilhar; e (3) que comunique informações de valor aos parceiros. Em suma, a percepção de prática de RSCORP provoca no profissional um sentimento de comprometimento e de confiança com a organização, favorecendo um sentimento de orgulho e pertencimento à organização (TURKER, 2009a; ALI et al., 2010; VLACHOS et al., 2010; PANAGOPOULOS et al., 2011; GLAVAS; KELLEY, 2014; YILMAZ; ALI; FLOURIS, 2015), diminuindo com isso, a IR. Nesse sentido, constatou-se que a adoção de práticas de RSCORP está associado a diminuição do pensamento, do planejamento e da vontade dos profissionais em deixarem suas organizações.

Foi observada a não rejeição da segunda e da terceira hipóteses de que as ações de responsabilidade social voltadas para os stakeholders sociais e não sociais (RSA) e voltadas para empregados (RSE) afetam positivamente a percepção de RSCORP. Alinhado com a perspectiva de Turker (2009a), os resultados sugerem que a percepção de ações organizacionais voltadas para a sociedade, meio ambiente, gerações futuras e organizações governamentais, bem como para a melhoria das condições de trabalho (tratamento justo da força de trabalho, carreira, educação, remuneração, incentivo ao voluntarismo, entre outros) exercem influência positiva sobre a RSCORP. Nesse ponto, observa-se também a relação de interdependência entre os fatores individuais e organizacionais que determinam a IR (JHA, 2009; HONGVICHIT, 2015).

Por fim, e de forma divergente a proposição de Turkey (2009a) a quarta e a quinta hipóteses de que as ações de responsabilidade voltadas para consumidores (RSC) e para governos (RSG) afetam positivamente a percepção de RSCORP foram rejeitadas. Os resultados indicam que as ações organizacionais voltadas para a proteção e para a satisfação de consumidores e que envolvam o cumprimento de aspectos legais nas relações com os governos não influenciam na percepção de RSCORP dos profissionais pesquisados.

\section{CONCLUSÕES}

O objetivo deste artigo foi o de analisar a relação existente entre a percepção das práticas de

RSCORP e a IR de profissionais brasileiros de diferentes Estados e setores econômicos. A 
conclusão central da pesquisa indica que quanto mais positiva for a percepção de práticas de RSCORP menor será a IR fazendo com que os profissionais diminuam o pensamento, o planejamento e a vontade de deixar suas organizações. Os resultados sugerem uma relação de interdependência entre os fatores individual e organizacional que determinam a IR. Esta interdependência leva o profissional a perceber que a adoção de práticas de RSCORP por parte de organização influencia o desenvolvimento de um sentimento de comprometimento e de confiança com a organização, diminuindo com isso, a IR

Os dados revelaram ainda que ações de responsabilidade social voltadas para os grupos de stakeholders sociais e não sociais e empregados afetam positivamente a percepção de práticas de responsabilidade social corporativa. Os resultados levam à rejeição das hipóteses de que as ações destinadas a consumidores e governo afetam positivamente esta percepção.

A retenção de profissionais nas organizações tem sido um tema que cada vez mais preocupam as organizações em função dos riscos e custos decorrentes desta perda de capital intelectual, fazendo com que os fatores que contribuem para a decisão de permanência na organização venham sendo estudados e planos de ação traçados. Entende-se, portanto, que se torna imperativo para as organizações identificar e compreender os motivos que desencadeiam a IR entre os seus empregados no cenário competitivo atual, de forma a promover ações que freiem este movimento visando alterar as políticas de RH, ainda sustentadas por práticas que não sejam mais suficientes para assegurar a retenção, permitindo ações preventivas. Segundo os resultados da pesquisa, uma das formas de se aumentar a percepção da RSCORP seria destacar os padrões legais e éticos dos produtos e serviços ofertados pelas organizações. Sendo assim, este estudo contribui para que as organizações busquem aperfeiçoar suas práticas de responsabilidade social destinada a diferentes grupos de stakeholders de forma a ampliar a percepção positiva e, consequentemente, ter profissionais que sintam orgulho de suas organizações, e por isso desenvolvam comportamentos positivos em relação a ela e não tenham interesse em deixá-la.

No âmbito acadêmico, o estudo contribui para aumentar o conhecimento que se tem sobre a RSCORP e seus impactos nas diversas ações estratégicas conduzidas pelas organizações, tema que ainda carece de amadurecimento. Além disso, aponta para a necessidade de novas pesquisas sobre os temas tratados e, particularmente, o desenvolvimento de escalas que permitam capturar com maior acurácia o entendimento sobre responsabilidade social corporativa e seus grupos de interesse, na medida em que os resultados demonstraram que 
algumas das variáveis utilizadas para capturar esta percepção não apresentaram o desempenho esperado.

Destaca-se como limitações da pesquisa o tamanho e caracterização da amostra e no corte transversal, devendo, portanto, ser replicada para uma população maior em um espaço de tempo superior. Um outro aspecto é que os profissionais foram tratados de forma homogênea na pesquisa, sendo relevante conduzir investigações de forma estratificada que permita captar as percepções de grupo heterogêneos de profissionais. Considerando a pequena quantidade de estudos brasileiros que tratem destas relações e considerando ainda, que o tema rotatividade tem sido cada vez mais discutido no âmbito das organizações, entende-se que novos estudos possam ser feitos para aumentar o conhecimento acumulado sobre o tema a partir de pesquisas empíricas considerando, em especial, a realidade de empresas brasileiras.

\section{REFERÊNCIAS}

ADEWALE, M. T.; RAHMON, T. A. Does corporate social responsibility improve an organization's financial performance? Evidence from Nigerian banking sector. IUP Journal of Corporate Governance, [S.1.], v. 13, n. 4, p. 52-60, 2014.

ALI, I.; REHMAN, K. U.; ALI, S. I.; YOUSAF, J.; ZIA, M. Corporate social responsibility influences, employee commitment and organizational performance. African Journal of Business Management, [S.1.], v. 4, n. 12, p. 2796-2801, 2010.

AGUINIS, H.; GLAVAS, A. What we know and don't know about corporate social responsibility: a review and research agenda. Journal of Management, Thousand Oaks, v. 38, n. 4, p. 932-968, 2012.

BASTOS, A. V. B. Comprometimento organizacional: um balanço dos resultados e desafios que cercam essa tradição de pesquisa. Revista de Administração de Empresas, São Paulo, v. 33, n. 3, p. 52-64, 1993.

BIDO, D. S.; SILVA, D.; SOUZA, C. A.; GODOY, A. S. Indicadores formativos na modelagem em equações estruturais com estimação via PLS-PM: como lidar com a multicolinearidade entre eles? In: ENCONTRO DE ENSINO E PESQUISA EM ADMINISTRAÇÃO E CONTABILIDADE, 2., 2009, Curitiba. Anais... Rio de Janeiro: ANPAD, 2009.

CARMO, G. do. Antecedentes da intenção de rotatividade: comprometimento organizacional e confiança do empregado na organização. 2009. 176 f. Dissertação (Mestrado em Psicologia Aplicada) - Programa de Pós-Graduação em Psicologia, Universidade Federal de Uberlândia, Uberlândia, 2009.

CARROLL, A. B. The pyramid of corporate social responsibility: toward the moral management of organizational stakeholders. Business Horizons, Bloomington, v. 34, n. 4, 
p. 39-48, 1991.

CARROLL, A. B. Corporate social responsibility: evolution of a definitional construct. Business \& Society, Thousand Oaks, v. 38, n. 3, p. 268-295, Sep. 1999.

CHANG, E. Career commitment as a complex moderator or organizational commitment and turnover intention. Human Relations, Thousand Oaks, v. 52, n. 10, p. 1257-1278, 1999.

COHEN, J. Statistical power analysis for the behavioral sciences. Mahwah, New Jersey: Lawrence Erlbaum, 1988.

COSTA, F. M.; BASTOS, A. V. B. Comprometimento organizacional: bases para uma abordagem processual. Psicologia; Teoria e Pesquisa, Brasília, v. 30, n. 3, p. 329-337, 2014.

ELLIS, A. D. The impact of corporate social responsibility on employee attitudes and behaviors. Academy of Management, Briarcliff Manor, v. 14, p. 1-6, 2009.

FREEMAN, R. E. Strategic management: a stakeholder approach. Boston: Pitman, 1984.

FRIEDMAN, M. The social responsibility of business is to increase its profits. The New York Times Magazine, New York, Sep. 1970.

GAUDÊNCIO, P.; COELHO, A.; RIBEIRO, N. Organisational CSR practices: employees' perceptions and impact on individual performance. International Journal of Innovation Management, [S.1.], v. 18, n. 4, p. 1-26, 2014.

GLAVAS, A.; KELLEY, K. The effects of perceived corporate social responsibility on employee attitudes. Business Ethics Quarterly, Cambridge, v. 24, n. 2, p. 165-202, 2014.

GREENING, D. W.; TURBAN, D. B. Corporate social performance as a competitive advantage in attracting quality workforce. Business and Society, Thousand Oaks, v. 39,n. 2, p. 254-280, 2000.

HAIR JR., J; HULT, G.; RINGLE, C.; SARSTEDT, M. A primer on partial least squares structural equation modeling (PLS-SEM). California: Sage publications, 2014.

HART, S. L.; MILSTEIN, M. B. Criando valor sustentável. RAE Executivo. Edição Especial, v. 3, n. 2, p. 65-79, maio/jul. 2004.

HAYES, B. E. Medindo a satisfação do cliente: desenvolvimento e uso de questionários. Rio de Janeiro: Qualitymark. 2001.

HELM, S. A matter of reputation and pride: associations between perceived external reputation, pride in membership, job satisfaction and turnover intentions. British Journal of Management, Hoboken, v. 24, n. 4, p. 542-556, 2013.

HERRMANN, K. Corporate social responsibility and sustainable development: the European Union initiative as a case study. Indiana Journal of Global Legal Studies, Bloomington, v. 11, n. 2, p. 205-232, 2004. 
HONGVICHIT, S. The research progress and prospect of employee turnover intention. International Business Research, [S.1.], v. 8, n. 6, p. 218-223, 2015.

JHA, S. Determinants of employee turnover intentions: a review. Management Today, Twickenham, v. 9, n. 2, p. 26-33, 2009.

KHAN, H. A. Impacts of corporate social responsibility on employees behavior in telecom sector of Pakistan. European Journal of Business and Management, Amsterdam, v. 6, n. 11, p. 34-43, 2014.

LONG, C. S.; THEAN, L. Y.; ISMAIL, V. K. V.; JUSOH, A. Leadership styles and employees' turnover intention: exploratory study of academic staff in a Malaysian College. World Applied Sciences Journal, [S.1.], v. 19, n. 4, p. 575-581, 2012.

MAIGNAN, Isabelle. Consumers' perceptions of corporate social responsibilities: a crosscultural comparison. Journal of Business Ethics, New York, v. 30, n. 1, p. 57-72, 2001.

MELO, D. C., OLIVEIRA, A. F. Intenção de rotatividade: impacto dos valores e do clima organizacional. In: REUNIÃO ANUAL DE PSICOLOGIA, 38., 2008, Uberlandia.

Resumos... Ribeirão Preto: SBP, 2008.

MOON, J. The contribution of corporate social responsibility to sustainable development. Sustainable Development, [S.1.], v. 15, n. 5, p. 296-306, 2007.

MORGAN, R. M.; HUNT, S. G. The commitment-trust theory of relationship marketing. Journal of Marketing, [S.1.], v. 58, n. 3, p. 20-38, 1994.

MOWDAY, R.; PORTER, L.; STEERS, R. Employee-organization linkages: the psychology of commitment, absenteeism, and turnover. New York: Academic Press, 1982.

OLIVEIRA, A. F. Confiança do empregado na organização: impacto dos valores pessoais, organizacionais e da justiça organizacional. 2004. 259 f. Tese (Doutorado em Psicologia). Universidade de Brasília, Brasília, 2004.

PANAGOPOULOS, N. G.; RAPP, A.; VLACHOS, P. A.; Corporate social performance and employees: construed perceptions, attributions and behavioral outcomes. SSRN Electronic Journal, [S.1.], nov. 2011. Disponível em: SSRN: <http://ssrn.com/abstract=1858084.>

PORTER, M. E.; KRAMER, M. The competitive advantage of corporate philanthropy. Harvard Business Review, Cambridge, Dec. 2002.

PORTER, M. E.; KRAMER, M. Strategy and society: the link between competitive advantage and corporate social responsibility. Harvard Business Review, Cambridge, dec. 2006.

POLIZZI FILHO, A.; SIQUEIRA, M. M. M. O impacto de bem-estar no trabalho e de capital psicológico sobre intenção de rotatividade: um estudo com professores. In: CONGRESSO NACIONAL DE PSICOLOGIA DA SAÚDE, 9., Aveiro, 2012. Anais... Lisboa: Placebo, 2012. 
RINGLE, C. M.; WENDE, S.; BECKER, J.M. SmartPLS 3. Hamburg, Germany: SmartPLS. Retrieved from www.smartpls.com. 2014.

RINGLE, C.; SILVA, D.; BIDO, D. S. Modelagem de equações estruturais com utilização do Smartpls. Revista Brasileira de Marketing, São Paulo, v. 13, n. 2, p. 54-71, 2014.

SCOTT, D. Retention of key talent and the role of rewards. WorldatWork Journal, [S.1.], v. 21, n. 4, p. 58-70, 2012.

SEN, S.; BHATTACHARYA, C. B. Does doing good always lead to doing better? Consumer reactions to corporate social responsibility. Journal of marketing Research, Chicago, v. 38, n. 2, p. 225-243, 2001.

SIQUEIRA, M. M. M.; GOMIDE JR., S.; OLIVEIRA A. de F. POLIZZI FILHO. A. Intenção de rotatividade. In: SIQUEIRA, M. M. M. (Org). Novas medidas do comportamento organizacional: ferramentas de diagnóstico e de gestão. Porto Alegre: Artmed, 2014. p. 209215.

TURKER, D. How corporate social responsibility influences organizational commitment. Journal of Business Ethics, New York, v. 89, n. 2, p. 189-204, 2009a.

TURKER, D. Measuring corporate social responsibility: a scale development study. Journal of Business Ethics, New York, v. 85, n. 4, p. 411-427, 2009b.

VASCONCELOS, K. C. de A., SILVA JUNIOR, A. da; SILVA, P. de O. M. da. Educação gerencial para atuação em ambientes de negócios sustentáveis: desafios e tendências de uma escola de negócios brasileira. Revista de Administração Mackenzie - RAM, São Paulo, v. 14, n. 4, 2013.

VLACHOS, P.A.; THEOTOKIS, A.; PANAGOPOULOS, N. G. Sales force reactions to corporate social responsibility: attributions, outcomes, and the mediating role of organizational trust. Industrial Marketing Management, Amsterdam, v. 39, n. 7, p. 1207$1218,2010$.

YIN-FAH, B. C.; FOON, Y. S.; CHEE-LEONG, L.; OSMAN, S. An exploratory study on turnover intention among private sector employees. International Journal of Business and Management, [S.1.], v. 5, n. 8, p. 57-64, 2010.

YOO, J.M.; CHON, M.I L. The effect of CSR on employees' turnover intention: comparative study on harmful and non-harmful industry. Indian Journal of Science and Technology, [S.1.], v. 8, n. 21, p. 1-5, 2015.

YOUNG, S. L.; MAKHIJA, M. V. Firms' corporate social responsibility behavior: an integration of institutional and profit maximization approaches. Journal of International Business Studies, New York, v. 45, n. 6, p. 670-698, 2014.

YILMAZ, A.K.; ALI, I.; FLOURIS T. The effect of corporate social responsibility on pride in membership, job satisfaction and employee engagement. British Journal of Economics, Management \& Trade, New Castle, v. 9, n. 4, p. 1-12, 2015. 